\title{
Instantons from Low Energy String Actions.
}

\author{
P. M. Saffin ${ }^{1 *} \&$ Anupam Mazumdar ${ }^{2 \dagger} \&$ E. J. Copeland ${ }^{1 \ddagger}$ \\ ${ }^{1}$ Centre for Theoretical Physics, University of Sussex, Falmer, Brighton, U.K. BN1 9QJ \\ ${ }^{2}$ Astronomy Centre, University of Sussex, Falmer, Brighton, U. K. BN1 9QJ
}

(August 10, 2018)

\begin{abstract}
We look for instanton solutions in a class of two scalar field gravity models, which includes the low energy string action in four dimensions. In models where the matter field has a potential with a false vacuum, we find that non-singular instantons exist as long as the Dilaton field found in string theory has a potential with a minimum, and provide an example of such an instanton. The class of singular instanton solutions are also examined, and we find that depending on the parameter values, the volume factor of the Euclidean region does not always vanish fast enough at the singularity to make the action finite.
\end{abstract}

PACS numbers: $98.80 . \mathrm{Cq}$

\section{INTRODUCTION}

In a fascinating letter, Hawking and Turok [1] have recently discovered a new instanton solution which they argue corresponds to the nucleation of a Universe undergoing a period of open inflation [2], independent of the need for a period of false vacuum dominated inflation. Although the interpretation of the result has been questioned by a number of authors [3, [4, the fact that the instanton solution exists has generated a great deal of excitement [5 [8].

The instantons, which are non trivial solutions to the euclidean equations of motion, are interpreted as the spontaneous quantum nucleation of a spacetime with some distribution of fields defined on it whose future evolution is determined by the analytic continuation of the euclidean solution. A natural definition of cosmic time coincides with moving from one hyperbolic spatial section to another which leads to the interpretation of the solution as an open universe emerging from quantum tunneling.

Previous studies of instanton solutions possessing either 0(4), 9] or 0(5), 10] invariances have assumed a constant gravitational coupling. Although today there exist tight constraints on the allowed spatial and temporal variation of Newtons constant 11, these constraints could have been relaxed in the past. In other words we know that Einstein's theory of gravity is an effective theory, and the correct theory could well have allowed for the evolution of the fundamental coupling constants. In this letter we treat the gravitational coupling to matter as one such dynamical variable. Such theories have been well studied [12] and are known as scalar-tensor theories. Among the variants is the Jordan, Brans and Dicke (JBD) theory [12] which treats the coupling of a scalar field to the metric as a constant. Perhaps of greater significance though is the fact that the gravitational lagrangian inspired by low energy effective action for bosonic string theory 13] can also be regarded as a variant, where here the coupling constant determines the strength between the dilaton and graviton degrees of freedom. It is certainly worth while investigating the role of the Dilaton field in determining the corresponding Instanton solutions.

In this letter, we shall show that the presence of a varying dilaton field, can lead to both singular and non-singular instantons. Moreover we shall see that depending on the parameters of the model, unless we invoke a potential in the dilaton sector the euclidean action may become divergent.

In $\S 2$ we consider the form of the gravitational action in the Einstein frame of reference (related to the string frame by a conformal transformation) and detail the type of solutions we shall be investigating, based on an ansatz for the metric and field distributions. We present an argument which describes how regular bubble solutions are expected to exist when the matter field possesses a metastable vacuum, and show that in this case the dilaton is required to have a potential of its own which has some minimum. In $\S 3$ we address the potentially singular behaviour of the instantons and show that the euclidean action can be well defined (for singular instantons) or divergent depending on the values of the parameters in the model being looked at. This is then discussed in the context of instantons arising out of string theory.

\footnotetext{
${ }^{*}$ E-mail: p.m.saffin@sussex.ac.uk

${ }^{\dagger}$ E-mail: anupamm@star.cpes.susx.ac.uk

${ }^{\ddagger}$ E-mail: E.J.Copeland@sussex.ac.uk
} 


\section{THE MODEL}

The starting point of our analysis will be the action given by

$$
S=\int d^{4} x \sqrt{-g}\left[-\frac{1}{2 \kappa^{2}} R+\frac{1}{2}(\nabla \phi)^{2}+\frac{1}{2} e^{-\gamma \kappa \phi}(\nabla \sigma)^{2}-e^{-\beta \kappa \phi} V(\sigma)-U(\phi)\right],
$$

where $\kappa^{2} \equiv 8 \pi G$ and $G$ is Newton's constant. By choosing specific values for $\beta$ and $\gamma$ we return to some more familiar models [14. In particular, $\phi$ is related to the Brans-Dicke field in the JBD model or the dilaton in the low energy, dimensionally reduced superstring case. Here we shall refer to $\phi$ as the dilaton and $\sigma$ as the matter field. As is common practice in finding instantons we restrict our attention to solutions of the euclidean equations of motion which have an $\mathrm{O}(4)$ symmetry. That is to say, we impose a euclidean metric of the form

$$
d s^{2}=d \xi^{2}+b(\xi)^{2}\left(d \psi^{2}+\sin ^{2}(\psi) d \Omega_{(2)}^{2}\right)
$$

and require that the fields $\phi$ and $\sigma$ depend only on the radial coordinate $\xi$. By doing this we will of course miss solutions, but this ansatz has the quality that instantons with this symmetry are expected to have the lowest euclidean action, although this has only been proved for the flat space case [9]. The field equations and gravitational constraint for the euclidean action are found to be

$$
\begin{aligned}
\phi^{\prime \prime}+3 \frac{b^{\prime}}{b} \phi^{\prime} & =-\frac{1}{2} \gamma e^{-\gamma \phi} \sigma^{2}-\beta e^{-\beta \phi} V(\sigma)+\frac{\partial U}{\partial \phi} \\
\sigma^{\prime \prime}+3 \frac{b^{\prime}}{b} \sigma^{\prime} & =\gamma \phi^{\prime} \sigma^{\prime}+e^{-(\beta-\gamma) \phi} \frac{\partial V}{\partial \sigma} \\
b^{\prime 2} & =1+\frac{1}{3} b^{2}\left[\frac{1}{2} \phi^{2}+\frac{1}{2} e^{-\gamma \phi} \sigma^{\prime 2}-e^{-\beta \phi} V(\sigma)-U(\phi)\right],
\end{aligned}
$$

where $\phi^{\prime} \equiv d \phi / d \xi$ etc, and we have rescaled the functions and coordinates by $\kappa$ to remove it from the problem. These equations may be used to generate the second order evolution equation for $b(\xi)$

$$
b^{\prime \prime} / b=-\frac{1}{3}\left[\phi^{\prime 2}+e^{-\gamma \phi} \sigma^{2}+e^{-\beta \phi} V(\sigma)+U(\phi)\right] .
$$

From this we see that $b(\xi)$ is convex as the right hand side of Eq. (6) is negative semi definite. Taking $b(\xi)$ as positive in the region of interest shows that it will vanish at two points, it then looks like a deformed sine function with a maxima at some $\xi_{\max }$, meaning that Eq. (2) represents a deformed four sphere. The vanishing of $b(\xi)$ then occurs at the poles of this deformed $S^{4}$ and are denoted by $\xi_{S}(=0)$ and $\xi_{N}$. If we want to find regular finite solutions to Eqs. (35) then the boundary conditions must be such that $\phi^{\prime}\left(\xi_{N}\right)=\phi^{\prime}\left(\xi_{S}\right)=0$ and $\sigma^{\prime}\left(\xi_{N}\right)=\sigma^{\prime}\left(\xi_{S}\right)=0$, otherwise the $b^{\prime} / b$ term will generate a divergent solution. Applying this condition to $\phi(\xi)$ we see immediately that without a dilaton potential, $U(\phi)$ the right hand side of Eq. (3) is negative semi-definite and so as we evolve from $\xi_{S}=0$ this drives $\phi\left(\xi \rightarrow \xi_{N}\right) \rightarrow-\infty$. The limiting behavior at this singular point leads to a curvature singularity and, depending on the value of $\beta$, can also cause the euclidean action to diverge unlike the instantons considered by Hawking and Turok [1]. If we are to find instantons with non singular profiles then we take $V(\sigma)$ to have a local minima at $\sigma=0$ and global one at $\sigma=\eta$. We have seen that $\phi$ must have a potential, but does this mean that if $U(\phi)$ exists we can find an instanton? Fortunately, as we will now show, $U(\phi)$ can be fairly generic. Following Coleman [15], we consider Eqs. (3 5) as representing two particles, $\Sigma$ and $\Phi$ with positions $\phi$ and $\sigma$ moving in their respective potential wells $-U(\phi)$ and $-V(\sigma)$ with some coupling to each other and a damping term $3 \frac{b^{\prime}}{b}$, which actually becomes anti damping when $\xi>\xi_{\max }$. The potentials that the particles move in are represented in figures 1 and 2 . The idea now is to find values for $\phi(\xi=0)$ and $\sigma(\xi=0)$ which will satisfy the boundary conditions at the poles. To analyze the problem we look for a self consistent set of profiles. Start by assuming that a regular profile exists for $\phi(\xi)$ and look at the matter field $\sigma$. The $\sigma$ 'particle', $\Sigma$ begins at $\sigma(\xi=0)=\sigma_{i}$ with zero velocity and it rolls down the potential $-V(\sigma)$. At $\xi=\xi_{N}$ it is required to have stopped, which will take an amount of time determined by the shape of the potential. If $\Sigma$ starts too close to $\sigma(\xi=0)=\eta$ then it can remain almost stationary up to $\xi_{\text {max }}$ when the anti damping term takes hold and pushes $\sigma$ to minus infinity, representing an overshoot solution. In order to make $\Sigma$ have a turn around point before $\xi_{N}$ then $\Sigma$ must start further from $\eta$, and if it starts just above $\sigma_{*}$ then it will turn around in a timescale governed by the harmonic oscillations due to $-V(\sigma)$ around $\sigma_{*}$. So in fact, depending on the nature of the potential, non trivial solutions may not exist if the harmonic oscillator timescale is longer than $\xi_{N}-\xi_{S}$, in which case the 
only instanton solution then available to us is the analogue of the Hawking Moss Instanton [10] where the fields are constant,

$$
\begin{aligned}
\sigma & =\sigma_{*} ; \\
\beta e^{-\beta \phi_{*} V}\left(\sigma_{*}\right) & =\left.\frac{\partial U}{\partial \phi}\right|_{\phi_{*}} ; \\
b(\xi) & =H^{-1} \sin (H \xi) ; \\
H^{2} & =\left[e^{-\beta \phi_{*}} V\left(\sigma_{*}\right)+U\left(\phi_{*}\right)\right] / 3 .
\end{aligned}
$$

In this case the metric represents that of an exact $S^{4}$, so that the symmetry of this solution is extended from the assumed $\mathrm{O}(4)$ to $\mathrm{O}(5)$. If we are in the situation where harmonic oscillations are faster than the $b(\xi)$ timescale then there will be a value, $\sigma_{*}<\sigma_{i}<\eta$, such that $\sigma^{\prime}\left(\xi_{N}\right)=0$. Turning our attention to the particle with position $\phi$, $\Phi$, we assume that $\sigma(\xi)$ has a regular form and then roll $\Phi$ in its background. The forcing terms for $\Phi$ on the right hand side of Eq. (3) consist of the first two terms driving $\Phi$ in the negative direction and a potential term which depends on the position of $\Phi$. If we consider the potential shown in figure 2 then by starting $\phi(\xi=0)=0$, all the terms force $\phi$ negative causing $\phi\left(\xi=\xi_{N}\right) \rightarrow-\infty$. This is the undershoot solution. By starting $\Phi$ sufficiently large and positive, the potential term can always dominate the other forces so that $\phi\left(\xi=\xi_{N}\right) \rightarrow+\infty$, the overshoot solution. It should then be clear that in between the overshoot and undershoot regimes there is a case where $\phi(\xi)$ remains finite. To obtain a qualitative picture of the form for $\phi(\xi)$ we note that if the negative forcing terms dominate at the start, they always dominate, so that initially we must have $\phi(\xi)$ increasing from $\phi_{i}$ through to some maxima where the negative driving terms finally become important and reduce $\phi(\xi)$ to end up at $\phi\left(\xi=\xi_{N}\right)=\phi_{f}$. We have numerically evolved the set of equations (3 6 ) for the specific choice of $\beta=2 \sqrt{2}, \gamma=\sqrt{2}$ (which corresponds to $\phi$ being the dilaton of the dimensionally reduced superstring action [14]) and a quadratic potential for $\phi$. The resulting profiles are shown in figure 3 and confirm the heuristic arguments given above. This is encouraging as it appears possible to obtain finite instanton solutions for a range of initial conditions arising from the low energy string action.

\section{FINITE AND SINGULAR EUCLIDEAN ACTIONS?}

One of the key results from Hawking and Turok was that the singular behavior of the tunneling matter field does not necessarily imply a divergent Euclidean action [1]. With this in mind we should not restrict our attention to finite Instanton configurations, and so we now consider the analogue singular solutions in the presence of the Dilaton. As we are no longer requiring regular field profiles then the potential for $\sigma$ no longer needs the rather specific form of a tunneling potential. In [1] the authors investigated a model which corresponds to Eq. (11) where $\phi=0$. They concluded that the resulting profiles for $\sigma$ and $b$ would have finite action, even though $\sigma$ itself diverges. This is because the volume factor $b^{3}(\xi)$ suppresses the divergences in the integrand. By keeping the $\phi$ field we have obtained numerical solutions to Eqs. (4-16) which can lead to either finite or divergent actions depending on the parameters $\beta$ and $\gamma$, a result which on the face of it seems to go against the spirit of the rather general arguments presented in [1]. However, our result can be traced back to the prescence of exponentials in the equations of motion. Indeed we can see how the inclusion of such terms in the model of [1] may also lead to a divergent action. To see this we drop $\phi$ from the model and consider the nature of the singularity at $\xi_{N}$. It is expected to be dominated by the derivatives of the profiles (backed up by numerical results), so that we quickly arrive at,

$$
\begin{aligned}
\sigma\left(\xi \rightarrow \xi_{N}\right) & \rightarrow \ln \left(\xi-\xi_{N}\right)^{\sqrt{2 / 3}} \\
b\left(\xi \rightarrow \xi_{N}\right) & \rightarrow\left(\xi-\xi_{N}\right)^{1 / 3} .
\end{aligned}
$$

The euclidean action is given by $S_{e}=-2 \pi^{2} \int d \xi b^{3}(\xi) V(\sigma)$, such that its integrand for an exponential potential $V(\sigma)=\exp (-A \sigma)$, rather than power law, becomes $\xi^{1-A \sqrt{2 / 3}}$. For this to be integrable we require $1-A \sqrt{2 / 3}>-1$, corresponding to $A<\sqrt{6}$. As mentioned earlier, a detailed numerical analysis of the system of evolution equations has borne out this inequality.

What is the potential significance of this result? In low energy effective actions of string theory, the dilaton naturally has an exponential type potential when non perturbative features such as gaugino condensates are included [16]. It could be that the only allowed instanton solutions (i.e. finite action) are those that correspond to non singular profiles, which in turn require the introduction of a tunneling field. An analysis of the singularity in the model with both a dilaton $\phi$ and matter field $\sigma$ leads to a similar conclusion only this time the inequalities for the constants $\beta$ and $\gamma$ depend on the nature of the potentials $V(\sigma), U(\phi)$. In particular, the values corresponding to the low energy 
superstring effective action of $\beta=2 \sqrt{2}$ and $\gamma=\beta / 2$ require a tunneling potential for $\sigma$ as the divergence of the profiles is too strong to be suppressed by the behaviour of the scale factor.

As far as the future evolution of the fields are concerned we do not consider the details here, they may be found elsewhere [1] [6] [17. The main principle to note is that the metric Eq. (2) can be analytically continued to a lorentzian metric, along a slice of the instanton where the metric is stationary, which then becomes the nucleated spacetime. The form of the spacetime clearly depends on how we choose to slice the instanton but we shall not consider this here, rather we refer the reader to the literature [1,5,6, 17. The analytically continued metric turns out to have singularities where the scale factor vanishes however, the only curvature singularity occurs for those cases where the north pole of the divergent instanton is included in the instanton slice. The other singularities of the metric turn out to be only coordinate singularities and the spacetime may be extended through them. One important aspect of this work is related to stabilizing the dilaton. If we are to take the value for $\beta$ and $\gamma$ as predicted by the low energy limit of superstring theory, then the singular instantons appear to be too singular to actually nucleate as their action diverges. This implies that we are only allowed the regular instantons which, by the above arguments, will lead to a dilaton profile nucleated near its minimum and allow for the dilaton to be stabilized with a finite vacuum expectation value.

\section{CONCLUSIONS}

We have looked at a model of gravity which contains both the low energy, dimensionally reduced superstring action and the JBD theory. We have found that in the Einstein frame, regular instanton profiles exist for a class of dilaton potentials which possess a minimum when the matter field has a tunneling potential. Moreover, if the dilaton has no potential this will lead to instantons with divergent profiles. The finiteness of the euclidean action of such solutions then depends on the parameters and potentials appearing in the specific model. The main interest of these results

lies in the superstring effective action, where a dilaton field arises naturally and the parameters $\alpha=\beta / 2=\sqrt{2}$ cause the euclidean action to diverge, an effect observed both numerically and using an analysis similar to the one given in the text. The outcome is a potentially new method of stabilising the dilaton.

\section{ACKNOWLEDGEMENT}

P.M.S. and E.J.C. are supported by PPARC fellowships, A.M. by Inlaks foundation and an ORS award. We thank Kei-ichi Maeda, Jim Lidsey, Andrew Liddle for discussions, and A.M. acknowledges use of the Starlink computer system at the University of Sussex. 


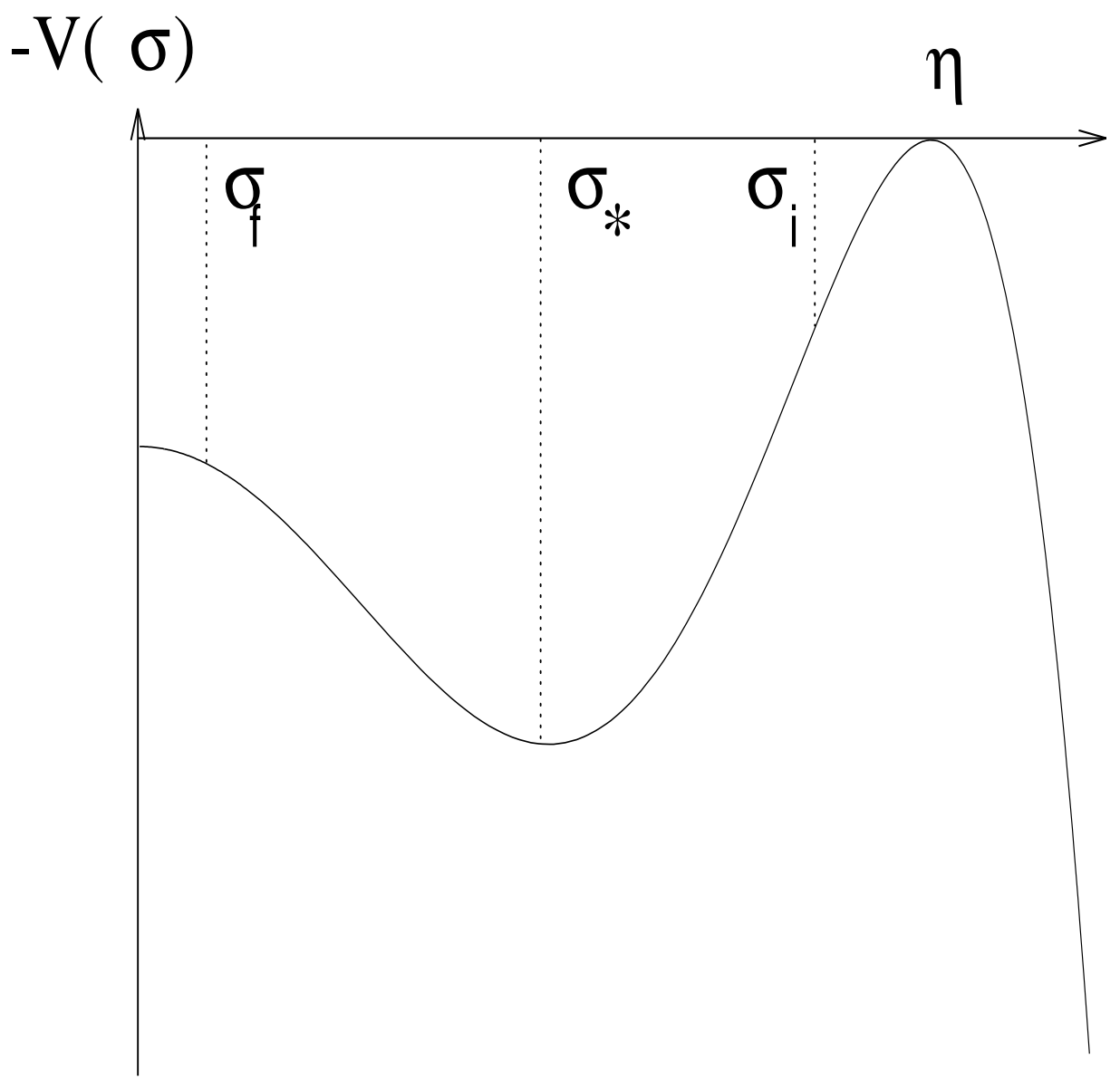

FIG. 1. $-\mathrm{V}(\sigma)$. 


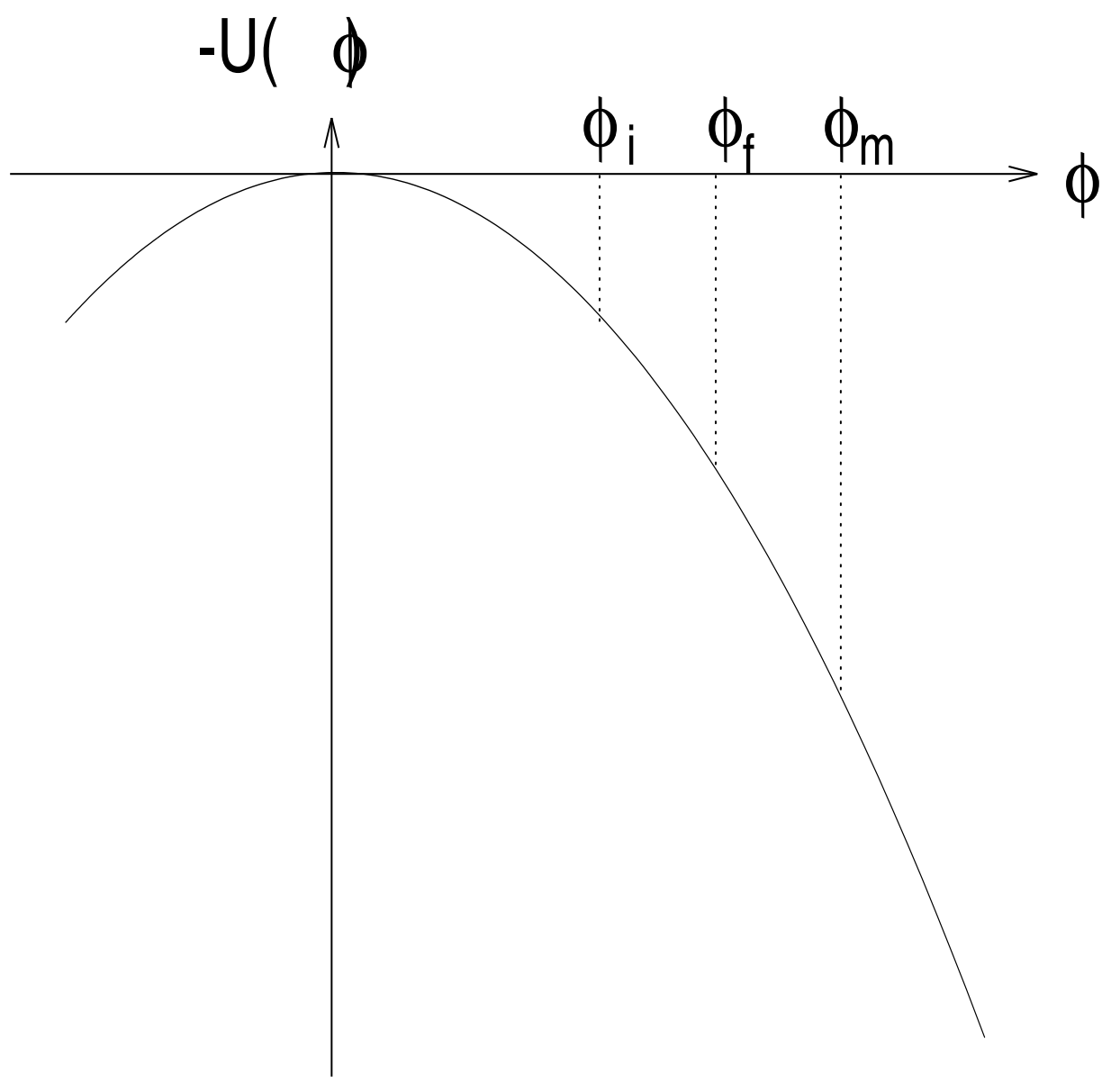

FIG. 2. $-\mathrm{U}(\phi)$. 


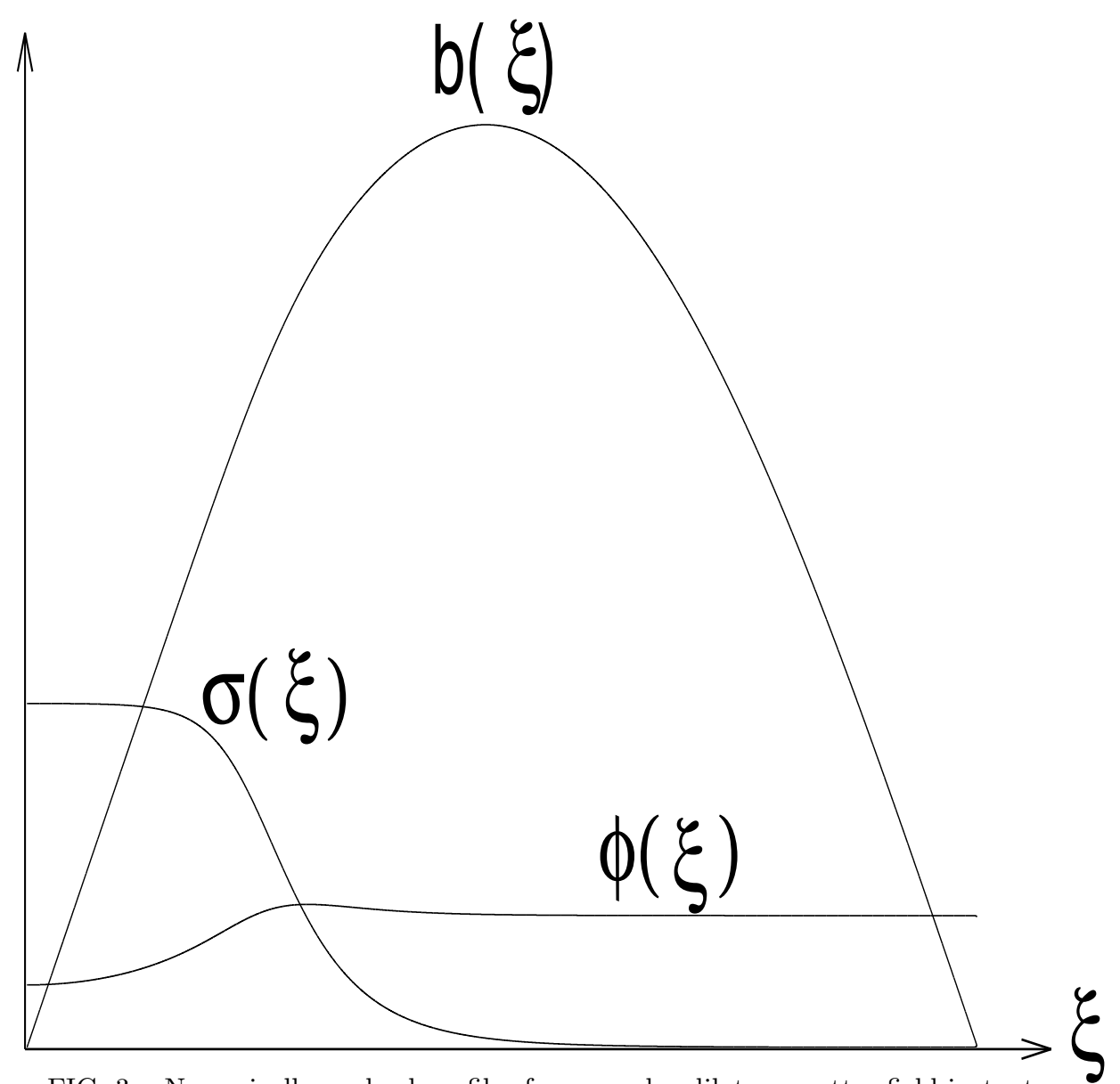

FIG. 3. Numerically evolved profiles for a regular dilaton, matter field instanton.

[1] S. W. Hawking and N. Turok, Open Inflation Without False Vacua, hep-th/9802030.

[2] J. R. Gott, Nature 295, 304 (1982); M. Bucher, A. S. Goldhaber and N. Turok, Phys. Rev. D 52, 3314 (1995); A. D. Linde, Phys. Lett. 351B, 99 (1995); A. D. Linde and A. Mezhlumian, Phys. Rev.D 52, 6789 (1995).

[3] A. Linde, Quantum Creation of an Open Inflationary Universe, gr-qc/9802038;

[4] A. Vilenkin, Singular instantons and creation of open universes, hep-th/9803084.

[5] R. Bousso and A. Linde, Quantum Creation of a Universe with $\Omega \neq 1$ : Singular and Non-Singular Instantons, grqc/980380638.

[6] W. G. Unruh, On the Hawking Turok solution to the Open Universe wave function, gr-qc/9803050.

[7] J. Garriga, Open inflation and the singular boundary, hep-th/9803210; Smooth "creation" of an open universe in five dimensions, hep-th/9804106.

[8] N. Turok and S. W. Hawking, Open Inflation, the Four Form and the Cosmological Constant, hep-th/9803156.

[9] S. Coleman and F. De Luccia, Phys. Rev.D 21, 3305 (1980).

[10] S. W. Hawking and I. G. Moss, Phys. Lett. 110 B, 35 (1982).

[11] C.M. Will, Theory and experiment in gravitational physics. CUP (1985).

[12] P. Jordan, Z. Phys. 157, 112 (1959); C. Brans and R. H. Dicke, Phys. Rev. 124, 925 (1961).

[13] E. Fradkin, Phys. Lett. 158 B 316 (1985); C. Callan, D. Friedan, E. Martinec and M. Perry, Nucl. Phys. B 262593 (1985); C. Lovelace, Nucl. Phys. B 273413 (1985).

[14] A. L. Berkin and K. Maeda, Phys. Rev. D 44, 1691 (1991).

[15] S. Coleman, Phys. Rev. D15, 2929 (1977).

[16] J.P. Derendinger, L.E. Ibáñez and H.P. Nilles, Phys. Lett. B155 65 (1985); M. Dine, R. Rohm, N. Seiberg and E. Witten, 
Phys. Lett. B156 55 (1985).

[17] A. H. Guth and E. J. Weinberg Nucl. Phys. B212 321 (1983), P. F. Gonzalez-Diaz hep-th/9805012.

\section{List of Figures}

\begin{tabular}{|c|c|c|c|}
\hline 1 & $-\mathrm{V}(\sigma)$ & & \\
\hline 2 & $-\mathrm{U}(\phi)$. & & 6 \\
\hline 3 & Numer & cally evolved profiles for a regular dilaton, matter field instanton. & 7 \\
\hline
\end{tabular}

Figure 1. - V $(\sigma)$. Figure 2. -U( $\phi)$. Figure 3. Numerically evolved profiles for a regular dilaton, matter field instanton. 